\title{
Evolution of the flaring active region NOAA 10540 as a sequence of nonlinear force-free field extrapolations
}

\author{
J. K. Thalmann and T. Wiegelmann
}

\author{
Max-Planck-Institut für Sonnensystemforschung, 37191 Katlenburg-Lindau, Germany \\ e-mail: [thalmann;wiegelmann] @mps.mpg.de
}

Received 4 February 2008 / Accepted 6 March 2008

\section{ABSTRACT}

\begin{abstract}
Context. The solar corona is structured by magnetic fields. As direct measurements of the coronal magnetic field are not routinely available, it is extrapolated from photospheric vector magnetograms. When magnetic flux emerges from below the solar surface and expands into the corona, the coronal magnetic field is destabilized, leading to explosive phenomena like flares or coronal mass ejections.

Aims. We study the temporal evolution of the flaring active region NOAA 10540 and are in particular interested in the free magnetic energy available to power the flares associated with it.

Methods. We extrapolated photospheric vector magnetograms measured with the Solar Flare Telescope, located in Tokyo, into the corona with the help of a nonlinear force-free field model. This coronal magnetic field model is based on a well-tested multigrid-like optimization code with which we were able to estimate the energy content of the 3D coronal field, as well as an upper limit for its free magnetic energy. Furthermore, the evolution of the energy density with height and time was studied.

Results. The coronal magnetic field energy in active region 10540 increases slowly during the three days before an M6.1 flare and drops significantly after it. We estimated the energy that was set free during this event as $\propto 10^{25} \mathrm{~J}$. A sequence of nonlinear force-free extrapolations of the coronal magnetic field shows a build up of magnetic energy before a flare and release of energy during the flare. The drop in magnetic energy of the active region is sufficient to power an M6.1 flare.
\end{abstract}

Key words. Sun: magnetic fields - Sun: flares - Sun: corona

\section{Introduction}

It is widely believed that the solar magnetic field is formed in the interface layer between the solar radiative and convective zones due to rapid changes in the rotation rate as one looks inward or outward across it. The field then emerges at the solar surface to form active regions (ARs), which are twisted by the Coriolis force. When magnetic flux emerges from below the solar surface and expands into the corona, the coronal magnetic field is destabilized, leading to explosive phenomena, such as coronal mass ejections (CMEs) and flares (see, e.g., Archontis et al. 2006). The free magnetic energy to be released during these events, is believed to be stored in current-carrying coronal magnetic fields that appear twisted or sheared. In general, the energy necessary for such large-scale phenomena is assumed to be stored and released in the corona, although the evolution of the lower lying photosphere certainly has an influence on the available amount of free energy above (see Barnes \& Leka 2006, and references therein).

How the photospheric fields are changing during flares or whether they play an even more active role in the course of solar flares is not clear (see, e.g., Kosovichev \& Zharkova 1999), since the fields are indeed measured at photospheric levels; but most of the energy is released in layers above the photosphere, for which hardly any direct measurements are available. On the one hand, Nakagawa (1976) suggests that the occurrence of flares simply manifests the passive response of local fields to large photospheric motions. More recently, Kosovichev \& Duvall (2006) also stress the importance of the connection of magnetic structures in the upper atmosphere with places near magnetic neutral lines in the photosphere, since permanent changes of the line-ofsight (LOS) component of the photospheric field clearly seem be related to the impulsive phase of flares. On the other hand, as described by Somov et al. (2002), photospheric reconnection is supposed to be responsible for supplying the higher layers with plasma, while its fast collisionless coronal counterpart can convert excess magnetic energy into kinetic or thermal energy to be released finally.

To investigate the magnetic field configuration in the solar corona, methods have been developed to derive it from routinely observed photospheric magnetograms. This is necessary, since direct measurements of the magnetic field vector in the chromosphere (e.g., Solanki et al. 2003) and corona (e.g., Lin et al. 2004) have only been done so far for a few individual cases.

The 3D coronal magnetic field structure is obtained by extrapolating measurements of the photospheric magnetic field vector into the corona. In general, existing extrapolation methods assume that the coronal magnetic field is in equilibrium without flows and that the coronal structures change on length scales comparable to the typical coronal scale height (Neukirch 2005). Furthermore, as the magnetic field is dominant in ARs, i.e., the magnetic pressure is higher than the plasma pressure, one can neglect nonmagnetic forces and assume that the coronal magnetic field is force-free. Mathematically, this can be expressed with

$(\nabla \times \boldsymbol{B}) \times \boldsymbol{B}=0$,

$\nabla \cdot \boldsymbol{B}=0$. 
Here (1) respresents the Lorentz force vanishing, which implies that the electric current density $\left(\mu_{0} \boldsymbol{j}=\nabla \times \boldsymbol{B}\right)$ is parallel to the magnetic field vector $(\boldsymbol{B})$, and (2) is the solenoidal condition. By introducing a scalar function (the sometimes so-called torsion function $\alpha$ ) in (1), these equations can be reformulated as

$\nabla \times \boldsymbol{B}=\alpha \boldsymbol{B}$,

$\boldsymbol{B} \cdot \nabla \alpha=0$,

where (4) is obtained by taking the divergence of (3) and by using (2), and it expresses that $\alpha$ is constant along the field lines. The upper equations describe (i) a potential field if $\alpha=0$; (ii) a linear force-free field if $\alpha=$ const. but non-zero; and (iii) a nonlinear force-free field if the torsion function is a function of space $(\alpha=f(\boldsymbol{r}))$. Different approaches are made to solve these potential (e.g., Schmidt 1964; Sakurai 1982) and linear forcefree (e.g., Chiu \& Hilton 1977; Seehafer 1978) boundary value problems. These extrapolation methods are mathematically and numerically easy to use and need only the LOS field component as input.

Coronal magnetic reconstruction techniques, mainly with the linear force-free assumption, have been used in many studies. For instance, van Driel-Gesztelyi et al. (2000) studied two sigmoid events related to CMEs by matching an extrapolated linear force-free field with Yohkoh/SXT (Ogawara et al. 1991; Tsuneta et al. 1991) data. Tang et al. (2000) used a trial and error method to fit a linear force-free model with Yohkoh/SXT and $\mathrm{H} \alpha$ data obtained with the Solar Flare Telescope in Tokyo (Sakurai et al. 1995) to study a brightening event. More recently, Mandrini et al. (2005) analyzed the coronal magnetic field around a sigmoidal X-ray bright point. In both cases, a fast Fourier transform method (originally proposed by Alissandrakis 1981) was used for extrapolating the linear force-free field from the photospheric LOS magnetic field component.

However, linear force-free models cannot recover the free magnetic energy to power eruptive processes as it is exactly the amount of energy that exceeds the energy of a linear force-free field that could partly be transformed into kinetic energy during dynamic events. Therefore, nonlinear force-free field models are needed to be able to describe the magnetic field behavior related to explosive events. Various methods have been developed to do this: e.g., Sakurai (1981), Wu et al. (1990), Amari et al. (1997, 1999), Wheatland et al. (2000), Régnier et al. (2002), Wiegelmann \& Neukirch (2003), Wheatland (2004), Wiegelmann (2004), Valori et al. (2005), Amari et al. (2006), Inhester \& Wiegelmann (2006), Song et al. (2006), Wheatland (2006), Yan \& Li (2006), Valori et al. (2007), Wheatland (2007), and Wiegelmann (2007). Some have been compared by Schrijver et al. (2006), Metcalf et al. (2008), and Schrijver et al. (2008). Another review of nonlinear force-free extrapolation methods is given by Wiegelmann (2008).

Using one or the other of the existing nonlinear force-free field extrapolation methods, several previous studies deal with the energy content of the magnetic field above solar ARs. For instance, Bleybel et al. (2002) used a Grad-Rubin-like nonlinear force-free field model to estimate the global energy budget of AR 7912 before and after a long duration C1.6 flare on 1995 October 14, finding a decrease in the magnetic energy over the course of the eruption. Both post- and pre-flare configurations showed a total magnetic energy content of $\propto 10^{25} \mathrm{~J}$.

Analyzing NOAA AR 8151 as visible in February 1998 by also using a Grad-Rubin-like method, Régnier et al. (2002) found that the available free magnetic energy was not high enough $\left(\propto 10^{24} \mathrm{~J}\right)$ to produce a large flare and concluded that this might be the reason why no EIT flare was observed. The 3D magnetic configuration of a simultaneously observed $\mathrm{H} \alpha$ filament and X-ray sigmoid has been studied in detail by Régnier \& Amari (2004). The same AR in its decaying phase was compared with the newly emerged 1998 May AR 8210 by Régnier \& Priest (2007b) who found the free energy of both ARs to be within the same order of magnitude. Furthermore, they concluded that this amount of energy was high enough to trigger a series of small flares that have been described in detail by Régnier \& Canfield (2006).

The same extrapolation method was used to analyze NOAA AR 10486 on 2003 October 27 and 28 by Régnier et al. (2005) where the amount of free energy was estimated as $\propto 10^{25} \mathrm{~J}$. The same AR on 2003 October 29 was explored by Metcalf et al. (2005) who found an increase in the free magnetic energy $\left(E_{\mathrm{free}} \propto 10^{26} \mathrm{~J}\right.$ as estimated by use of the magnetic virial theorem), which was available to be released during a X-class flare event.

Also, Régnier \& Priest (2007a) compared the free magnetic energy of various ARs at distinct evolutionary stages as computed by using different mathematical approaches. Most recently, Jing et al. (2008) have calculated the shear parameters around the flaring polarity inversion line of NOAA AR 10930 before and after an occurring X3.4 flare based on the extrapolation of Hinode/SOT (Kosugi et al. 2007) observations. Besides this, various nonlinear force-free extrapolation codes were applied to the pre- and post-flare Hinode/SOT data finding a decrease in the free magnetic energy of $3 \times 10^{25} \mathrm{~J}$, high enough to power an X-class flare (see Schrijver et al. 2008, and references therein).

Complementary to investigations with the help of the reconstructed 3D coronal magnetic fields are studies of photospheric vector magnetograms from, e.g., the Solar Flare Telescope (SFT) of the National Astronomical Observatory of Japan (NAOJ) in Mitaka, Tokyo. Several studies deal with the transport of magnetic helicity and Poynting flux across the photosphere, such as those carried out by Kusano et al. (2002) for NOAA AR 8100 between 1997 November 1 and 5, applying a local correlation technique. They found that horizontal shear and vertical motions are responsible for the helicity injection in a comparable amount and that the energy flux is mainly due to upwelling motions through the photosphere. Yamamoto et al. (2005) investigated the relation between magnetic helicity input and the structure of sigmoidal coronal loops for several ARs. Both sigmoidal and non-sigmoidal regions seem to have comparable helicity injection rates. In a statistical study, Maeshiro et al. (2005) inspected the correlation between helicity injection and soft X-ray activity with the help of NAOJ/SFT and Yohkoh/SXT data for seven ARs, finding a comparable amount of positive and negative magnetic helicity injections. Nindos et al. (2003) and Nindos $\&$ Andrews (2004) investigated the association of the magnetic helicity budget in ARs with big flares and CMEs by analyzing the helicity transport through the photosphere with best-fit linear force-free models. Even though a positive correlation was found, the helicity budget was underestimated by a factor of 2.9-4, which is assumed to be a consequence of using a linear forcefree, rather than a nonlinear force-free coronal magnetic field model.

We outline the paper as follows. In Sect. 2 we describe the method of extrapolation, Sect. 3 gives a brief description of the studied AR and the data set used, Sect. 4 shows the results gained from the nonlinear force-free field extrapolation, and conclusions are drawn in Sect. 5. 


\section{Method}

As the observed photospheric vector magnetogram data is performed from magnetic field measurements at a level in the solar atmosphere that is known to be not force-free, the solution to the force-free Eqs. (1) and (2) may not exist (see, e.g., Gary 2001; Metcalf et al. 1995). The consistency of the observed data with the force-free assumption can be checked with integral relations (see Molodenskii 1969; Molodensky 1974; and Aly 1989, for details). These criteria allow dimensionless parameters to be computed that represent a measurement of the total force and torque on the bottom boundary layer, i.e., the observed vector magnetograph data. They should be small in order to find a force-free solution in the corona above the observed magnetic field. To achieve the consistency of the observed photospheric data with the force-free assumptions, we apply a preprocessing routine as developed by Wiegelmann et al. (2006b). The preprocessed data provide consistent boundary conditions for a non-linear forcefree coronal magnetic field extrapolation. For an alternative preprocessing routine based on simulated annealing see Fuhrmann et al. (2007).

For extrapolating the nonlinear force-free coronal magnetic field from these more suitable boundary conditions, we use the multigrid-like optimization code of Wiegelmann (2004), which is a modification of the optimization approach originally proposed by Wheatland et al. (2000). It evolves the magnetic field matching the boundary, force-free, and divergence-free conditions by minimizing a volume integral of the form

$L=\int_{V} w(x, y, z)\left[B^{-2}|(\nabla \times \boldsymbol{B}) \times \boldsymbol{B}|^{2}+|\nabla \cdot \boldsymbol{B}|^{2}\right] \mathrm{d}^{3} x$.

This method has been intensively tested with several semianalytic and numerically created model ARs in Schrijver et al. (2006), Wiegelmann et al. (2006a), and Metcalf et al. (2008), and has previously been applied to extrapolate vector magnetograph data in Wiegelmann et al. (2005a), Wiegelmann et al. (2005b), Schrijver et al. (2008), and Jing et al. (2008).

Besides computing nonlinear force-free fields, we also calculated the corresponding potential fields that can be determined from the vertical photospheric field alone, using a Green's function method (see Aly 1989, and references therein). From these extrapolated 3D magnetic fields, we can estimate the total magnetic energy by calculating

$E=\frac{1}{2 \mu_{0}} \int_{V} B^{2} \mathrm{~d}^{3} x$,

where $\mu_{0}$ denotes the magnetic permeability of free space and $V$ the volume of the computational box. In the following, we denote the calculated total magnetic energy of the potential and nonlinear force-free field as $E_{\text {pot }}$ and $E_{\text {nlff }}$, respectively. Now, one can also roughly estimate an upper limit for the disposal of energy associated with coronal currents in the form

$E_{\text {free }}=E_{\text {nlff }}-E_{\text {pot }}$,

which is available to be released during dynamic explosive events. One can also estimate the energy density, i.e., the amount of energy stored in the given region of space per unit volume, by calculating

$u=\frac{B_{\mathrm{nlff}}^{2}}{2 \mu_{0}}$,

for a subvolume of the extrapolation domain which covers the flaring region.

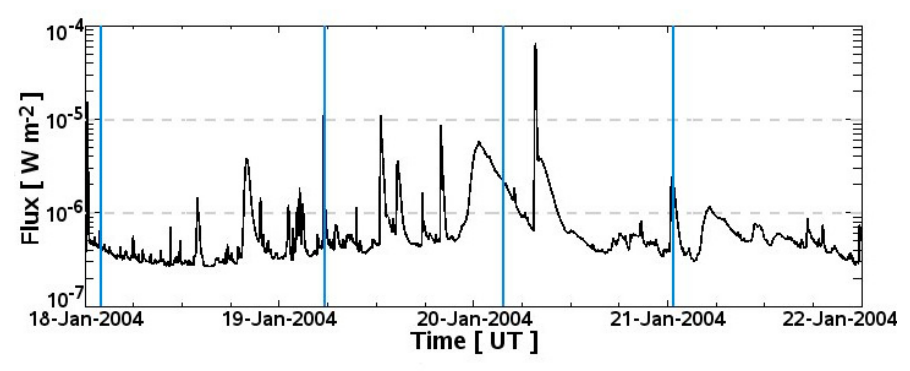

Fig. 1. Solar soft X-ray flux from Jan. 18-21, 2004, integrated in the wavelength range of $1-8 \AA$. Blue lines indicate when NAOJ/SFT data was available to use.

\section{Data}

\subsection{Flare activity of NOAA AR 10540}

According to the weekly bulletin of the Solar Influence Data Analysis Centers' (SIDC) Space Weather Application Pilot Project (SWAPP), the flaring activity during the week of January 12-16, 2004 was low, except for a C3.2 flare showing up on Jan. 15. Afterwards, the activity increased due to AR 10540 showing more peaks in the C-level and finally producing an M5.0 flare on Jan. 17 and an M1.4 flare on Jan. 18. The next week, this AR produced a long duration C8.2 flare on Jan. 19 accompanied by a full halo CME. On Jan. 21 two big prominence eruptions occurred, each leading to a CME, and after that the solar activity dropped to background B-level (see Fig. 1 for the solar soft X-ray flux from Jan. 18-22, 2004). Two M-class flares launched by AR 10540 are of special interest for the present study. The first, on Jan. 19 at 12:40 UT, was classified as an M1.0 flare and the second event, at 07:43 UT on Jan. 20, as an M6.1 flare. Besides this, a CME showed up first around 08:06 UT.

For nonlinear force-free field extrapolations, vector magnetograph data are needed near the solar disk center to minimize unavoidable foreshortening effects. If one wants to consider the temporal evolution of the coronal field, this of course represents a kind of restriction, since the observed AR follows the solar rotation. Nevertheless, the observation of the same AR for several consecutive days is important for making conclusions about the energy evolution before and after eventual eruptive phenomena. In the present study we investigate AR 10540 on four subsequent days (Jan. 18-21, 2004) at locations that are far enough from the solar limb (see Fig. 2).

\subsection{NAOJ/SFT vector magnetograph}

The Solar Flare Telescope (SFT) of the National Astronomical Observatory of Japan (NAOJ) consists of four telescopes, from which one, a vector magnetograph (VM, $20 \mathrm{~cm}$ aperture), measures the magnetic field vector by using the Zeeman effect on the Fe $6303 \AA$ spectral line, for which a narrow-band birefringent filter is centered at $6302.5 \AA$ (Sakurai et al. 1995). The data used here contain a region of $\approx 336^{\prime \prime} \times 315^{\prime \prime}$, i.e., $\approx 241 \mathrm{Mm} \times 226 \mathrm{Mm}$ (Fig. 3). We display the SFT field-of-view (FOV) on SoHO/MDI (Scherrer et al. 1995) LOS magnetograms nearest in time in Fig. 2. 

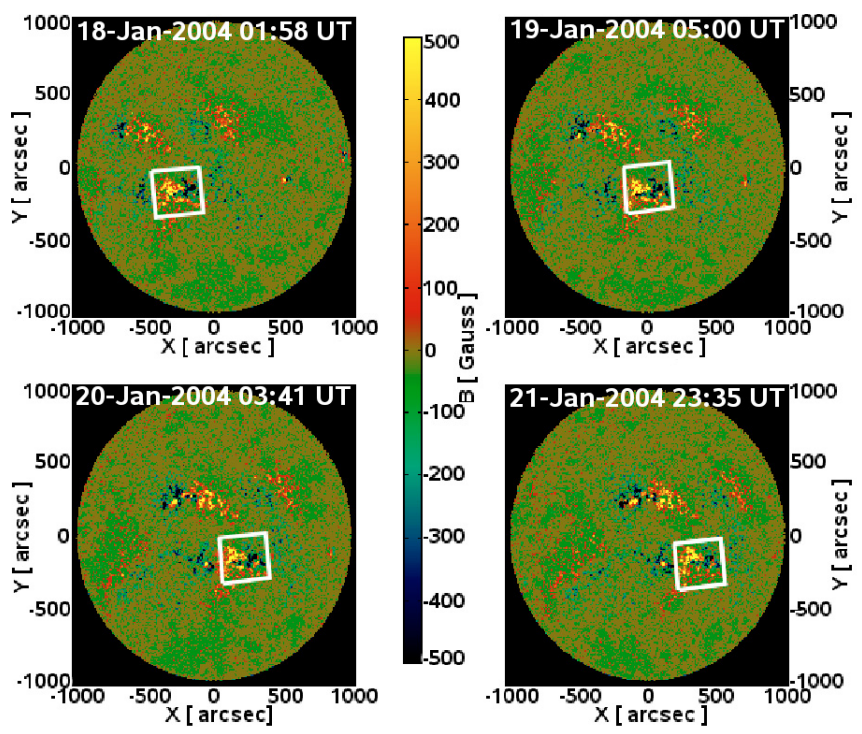

Fig. 2. SoHO/MDI LOS magnetograms from 2004 Jan. 18 to 21. White squares outline the NAOJ/SFT FOV, units are arcseconds from Sun center, and the colorbar indicates the field strength in Gauss.
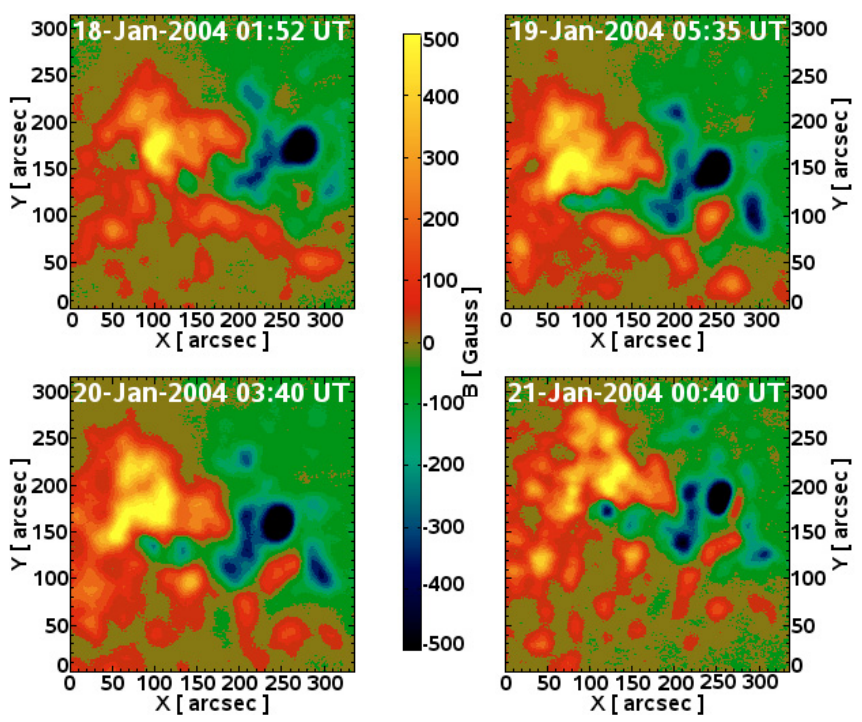

Fig. 3. LOS component of the NAOJ/SFT vector magnetograms from 2004 Jan. 18 to 21 . Units are arcseconds and the colorbar indicates the magnetic field strength in Gauss.

\section{Results}

\subsection{Global magnetic energy budget}

Using the observed SFT vector magnetograms as input data for our analysis, applying the preprocessing routine, and extrapolating the fields together gives us a set of potential and nonlinear force-free equilibria of the 3D configuration of coronal magnetic field above AR 10540, and we can calculate its magnetic energy content according to (6).

One finds that $E_{\text {nlff }}>E_{\text {pot }}$ (see Table 1), which shows that the potential field contains less magnetic energy than the nonlinear force-free field. The magnetic energy of the potential and nonlinear force-free field is slowly built up before the eruptive events and in the order of $E_{\mathrm{pot}}, E_{\mathrm{nlff}} \propto 10^{26} \mathrm{~J}$. However, all the energies go down remarkably after the M6.1 flare on Jan. 20 (see Fig. 4), whereas the M1.0 flare on Jan. 19 does not seem to have a significant effect on the energy content of the coronal field. This
Table 1. Total magnetic energy of the potential $\left(E_{\mathrm{pot}}\right)$ and nonlinear force-free $\left(E_{\mathrm{nlff}}\right)$ field as calculated from the extrapolated force-free field. $E_{\text {free }}=E_{\text {nlff }}-E_{\text {pot }}$ gives an upper limit for the free magnetic energy and $E_{\mathrm{nlff}} / E_{\mathrm{pot}}$ indicates at which amount the field relaxes towards the potential state.

\begin{tabular}{ccccc}
\hline \hline Date & \multicolumn{4}{c}{ Total magnetic energy $\left[\times 10^{26} \mathrm{~J}\right]$} \\
$(2004)$ & $E_{\text {pot }}$ & $E_{\text {nlff }}$ & $E_{\text {free }}$ & $E_{\text {nlff }} / E_{\text {pot }}$ \\
\hline Jan. 18 & 0.80 & 1.18 & 0.37 & 1.46 \\
Jan. 19 & 1.19 & 1.94 & 0.71 & 1.57 \\
Jan. 20 & 1.27 & 2.20 & 0.87 & 1.66 \\
Jan. 21 & 0.86 & 1.26 & 0.38 & 1.42 \\
\hline
\end{tabular}

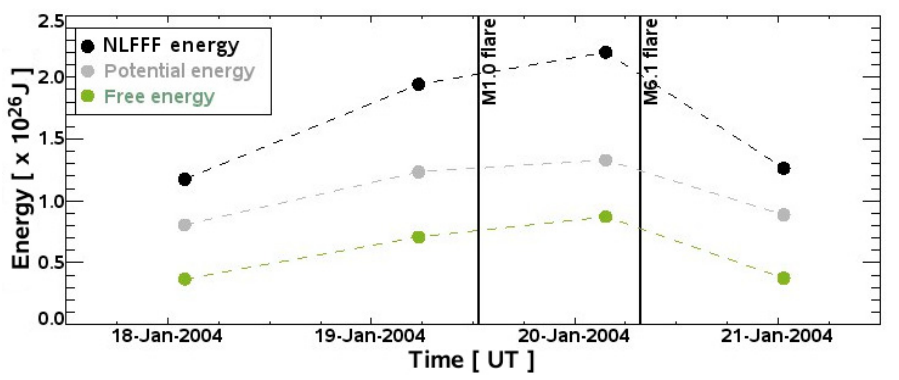

Fig. 4. Total magnetic energy of the nonlinear force-free field (top black curve), the potential field (middle gray curve), and an upper limit for the free magnetic energy (bottom green curve) on Jan. 18-21, 2004. Black lines indicate the two M-class flares.

impression may be due to the low time cadence of the available data (one vector magnetogram per day), as the influence of the M1.0 flare may simply not have been temporarily resolved.

We also estimate the ratio of the nonlinear force-free and potential fields' energy content $\left(E_{\mathrm{nlff}} / E_{\mathrm{pot}}\right.$ in Table 1$)$ as a relative measure of the available free energy normalized to the potential field (Fig. 5). Again, we find an increasing trend before the time of the larger M6.1 flare. A maximum of $E_{\mathrm{nlff}} / E_{\mathrm{pot}}=1.66$ is reached before the flare and drops to $E_{\mathrm{nlff}} / E_{\mathrm{pot}}=1.42$ after it. In other words, the magnetic field configuration is farthest away from the potential configuration before the occurrence of the M6.1 flare and is closer to it after releasing free magnetic energy during the explosion. However, the magnetic field does not relax to the potential field configuration, as this ratio does not reach unity; i.e., the whole amount of available magnetic energy is not set free during the M6.1 flare. Again, the M1.0 flare does not seem to contribute to a relaxation of the nonlinear forcefree towards the potential configuration. In the period of the occurrence of this smaller flare, magnetic flux was still emerging, hence suppressing the influence of the energy release even more during the M1.0 flare.

The excess energy of a force-free field over the corresponding potential field is an upper limit for the free magnetic energy available for dynamic processes that can be transformed into kinetic energy during flares or CMEs. We find an upper limit for the energy to be set free in the order of $E_{\text {free }} \propto 10^{25} \mathrm{~J}$ for all days, where again the largest amount of free energy $\left(E_{\text {free }} \approx 8.7 \times 10^{25} \mathrm{~J}\right.$ ) appears before the occurrence of the M6.1 flare and drops to $E_{\text {free }} \approx 3.8 \times 10^{25} \mathrm{~J}$ afterwards.

\subsection{Distribution of the energy density}

According to Goff et al. (2007), the M6.1 flare event showed a rising emission after $\sim 07: 32 \mathrm{UT}$, peaking twice at $\sim 07: 37 \mathrm{UT}$ and $\sim 07: 43$ UT, and finally decayed until 08:00 UT, followed by 


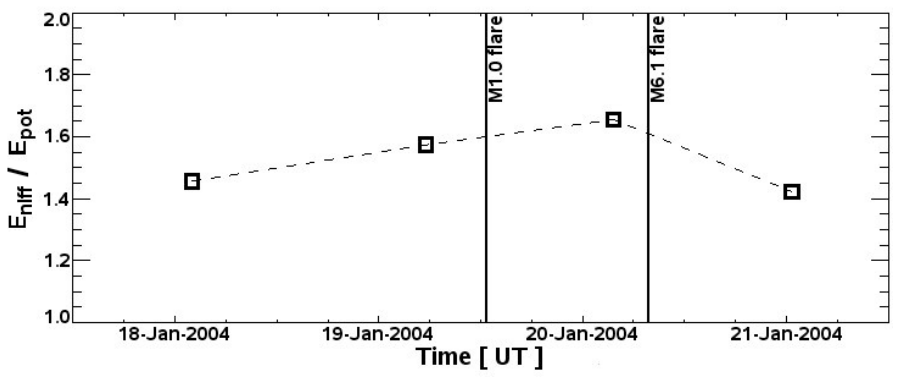

Fig. 5. Relative energies $\left(E_{\mathrm{nlff}} / E_{\mathrm{pot}}\right)$ for 2004 Jan. 18-21. Black lines indicate the two M-class flares.
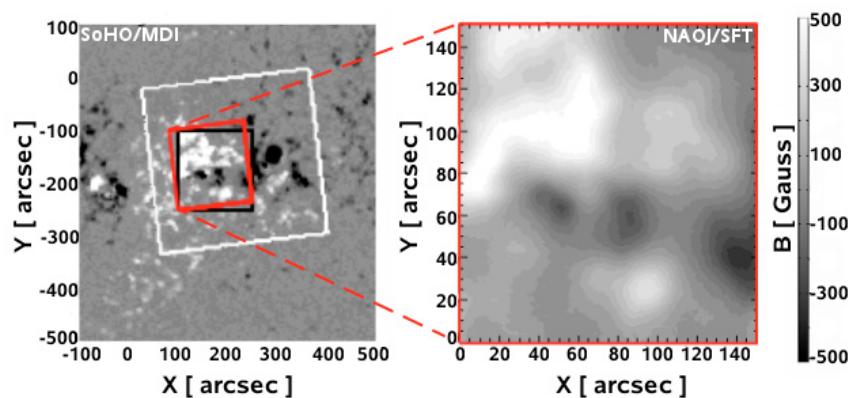

Fig. 6. MDI/LOS magnetogram on 2004 Jan. 20. The white square shows the SFT FOV. The black outline shows the subfield containing the flaring activity as visible in TRACE images. The corresponding subregion in the SFT image is outlined in red and shown in the right panel. Units are arcseconds and the colorbar shows the field strength in Gauss.

the first appearance of a CME around 08:06 UT. Backward extrapolation of the CME projected distance-time profile suggests a starting time between 07:10 UT and 07:25 UT, i.e., most probably launched even before the beginning of the observed M6.1 flare. They also performed a linear force-free magnetic field extrapolation based on SoHO/MDI data to outline the coronal field configuration on Jan. 20, and in the following we take a closer look at the temporal evolution of the energy density $(u)$ related to the flaring area as described in the mentioned study above. A subregion of the SFT data (Fig. 6) was selected due to the visibility of the flaring activity related to the M6.1 flare in TRACE (Handy et al. 1999) images.

According to the SFT subfield, we then extracted a subvolume of the 3D nonlinear force-free extrapolation domain of $\approx 150^{\prime \prime} \times 150^{\prime \prime} \times 75^{\prime \prime}(\approx 108 \times 108 \times 54 \mathrm{Mm})$ in $x, y$, and $z$ and estimated the integrated energy density $(e)$ as a function of height,

$e=\int_{S} u \mathrm{~d} x \mathrm{~d} y$

where $S$ denotes the $(x, y)$-plane at each height $z$ and also calculated the change in the energy density $(\Delta u)$ as

$\Delta u=u_{i+1}-u_{i}$,

where $u$ represents the energy density as defined in (8), $u_{i}$ denotes the energy density of the previous, and $u_{i+1}$ that of the following day. In this way we get insight into how energy gains or losses are distributed in the flaring region.

First, we integrated the energy density with the help of (9) and display it as a function of height in the subvolume, as shown in Fig. 7. One recognizes, that highest energy densities appear on Jan. $19\left(e_{19}\right)$ and $20\left(e_{20}\right)$. As expected, it seems that the main energy storage takes place before the occurrence of the M6.1 flare on Jan. 20 (i.e., $e_{21}<e_{19}, e_{20}$ ). After this flare was launched, the

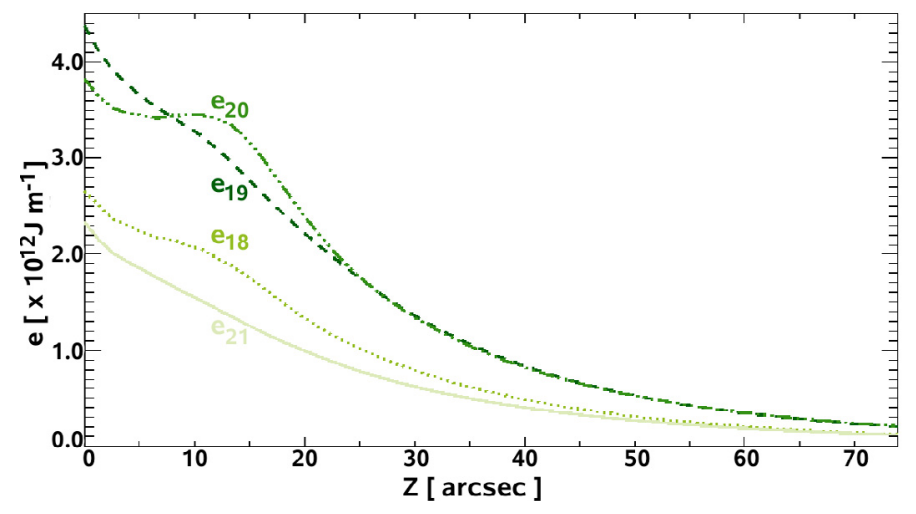

Fig. 7. Integrated energy density $(e)$ as a function of height in the subvolumes. $e_{18}$ (dotted), $e_{19}$ (dashed), $e_{20}$ (dashed dotted), and $e_{21}$ (solid) represent the integrated energy density as calculated for the subvolume of Jan. 18, 19, 20, and 21, respectively.

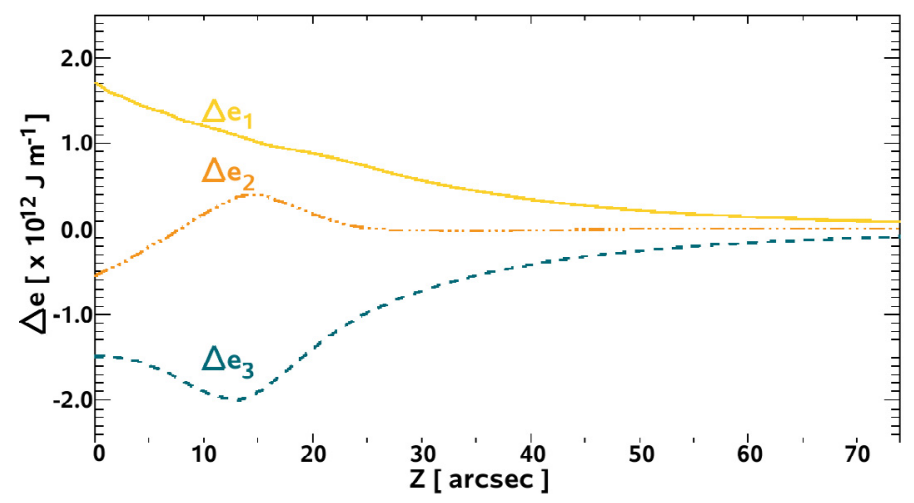

Fig. 8. Change in the energy density $(\Delta e)$ as a function of height in the subvolumes. $\Delta e_{1}=e_{19}-e_{18}, \Delta e_{2}=e_{20}-e_{19}$, and $\Delta e_{3}=e_{21}-e_{20}$ are represented by the solid, dashed dotted, and dashed lines, respectively.

amount of energy stored goes down remarkably. Besides this, the energy density on Jan. 18 is higher than on Jan. 21 (i.e., $\left.e_{21}<e_{18}\right)$ as well, which means that also on the first of the considered days the amount of stored energy is higher than after the M6.1 flare, which occurred on the last day of the data set. Nevertheless, not as much energy is available on Jan. 18 to power an explosive event as on Jan. 19 and 20 (i.e., $e_{19}, e_{20}>e_{18}$ ). Especially at the height of $\approx 10^{\prime \prime}-20^{\prime \prime}(\approx 7-14 \mathrm{Mm})$, even more energy was stored on Jan. 20 than on Jan. 19 (i.e., $e_{20}>e_{19}$ in this narrow height range of the subvolume).

Second, we investigated the change of the integrated energy density as a function of height within the subvolume by simply calculating $\Delta e_{1}=e_{19}-e_{18}, \Delta e_{2}=e_{20}-e_{19}$, and $\Delta e_{3}=e_{21}-e_{20}$ (see Fig. 8). One can see that $\Delta e_{1}$ and $\Delta e_{2}$ are mainly positive (i.e., $e_{19}>e_{18}$ and $e_{20}>e_{19}$, except that $\Delta e_{2}$ is negative at very low heights of $\lesssim 8^{\prime \prime}$, i.e., $\$ 6 \mathrm{Mm}$ above the photosphere), whereas $\Delta e_{3}$ is mainly negative (i.e., $e_{21}<e_{20}$ ). However, as is also clearly visible in Figs. 7 and 8, the absolute values of the integrated energy density decrease with increasing height. It can not be concluded without some doubt that the temporal evolution of the integrated energy density is purely a consequence of the flaring activity, since until Jan. 20 magnetic flux was still emerging in this AR.

Finally, we show the total magnetic energy content as calculated from the nonlinear force-free field, together with the change in the energy density $(\Delta u)$ in the extracted subvolumes in Fig. 9. Panel (a) shows $\Delta u_{1}=u_{19}-u_{18}, \Delta u_{2}=u_{20}-u_{19}$ is shown in panel (b), and $\Delta u_{3}=u_{21}-u_{20}$ in panel (c). As found 

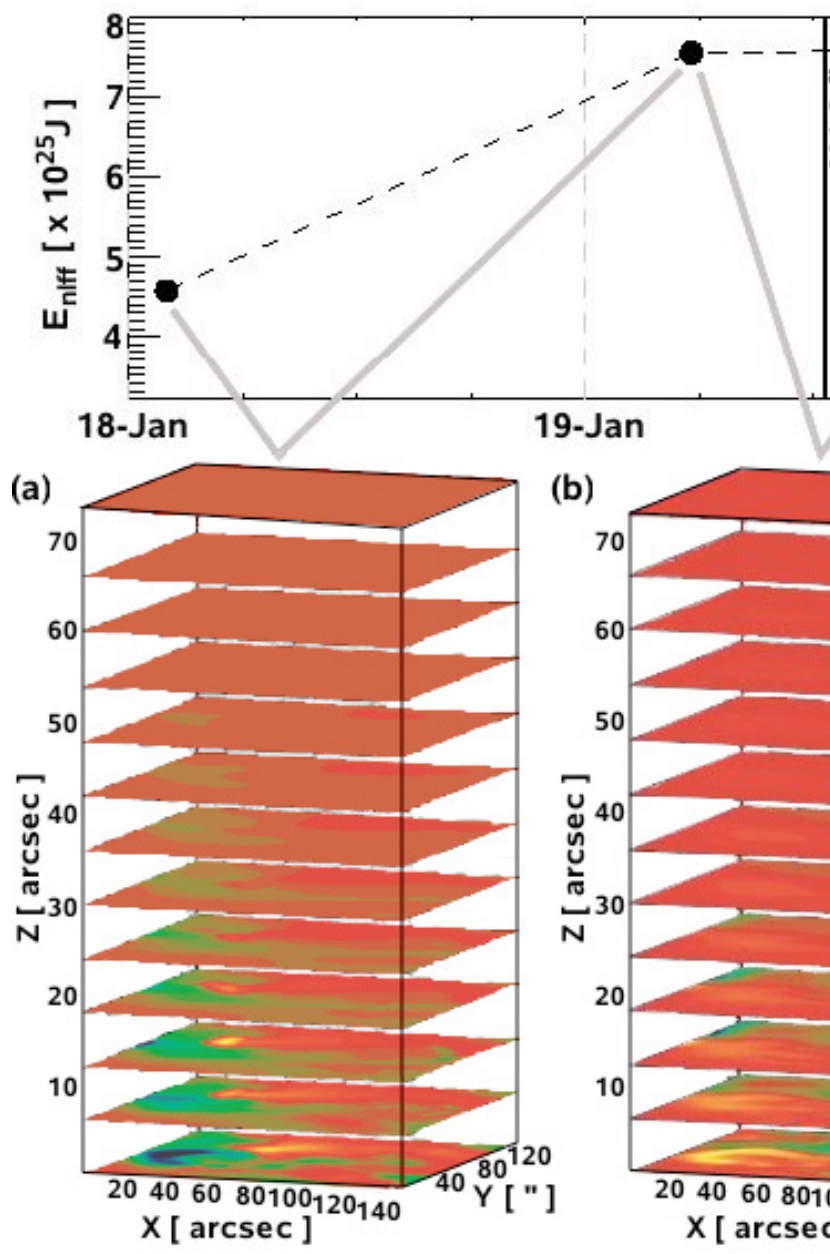

(b)

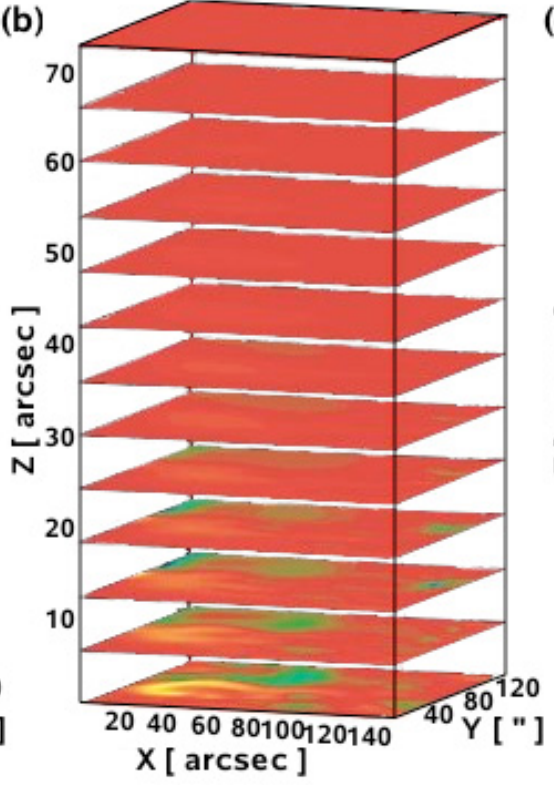

$\mathrm{X}[\operatorname{arcsec}]$

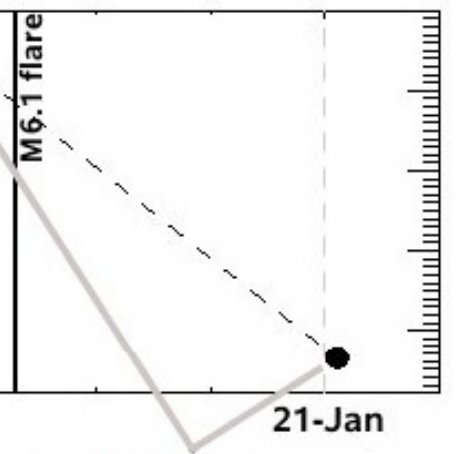

(c)

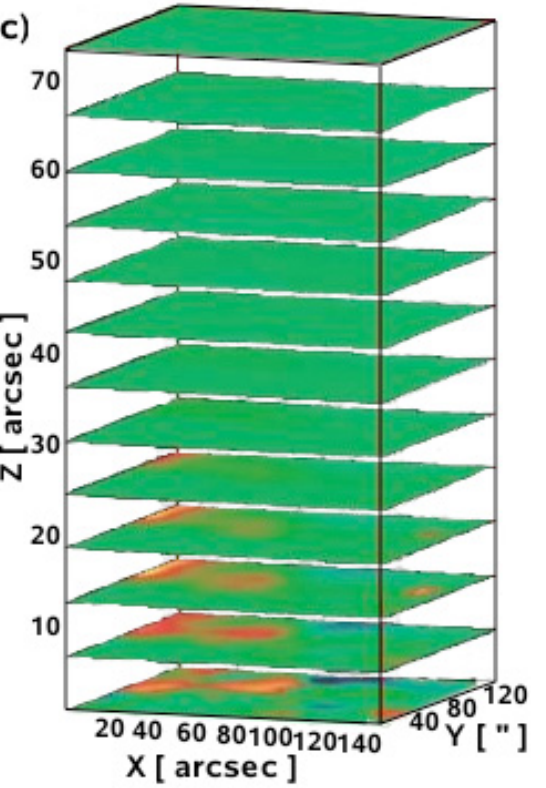

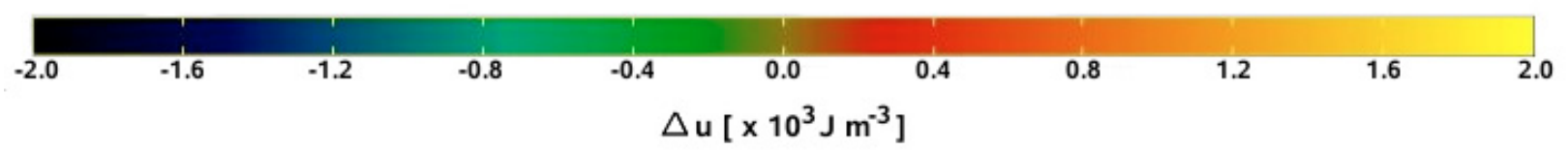

Fig. 9. Upper panel: total magnetic energy of the nonlinear force-free field for the chosen subvolumes for the consecutive days. The lower three panels show the difference in the energy density $(\Delta u)$ between the consecutive days in the subvolume. For better visibility, the $z$-axis is drawn elongated. Color-coded in the subvolumes is the change of the energy density between Jan. 18 and 19 in panel a), between Jan. 19 and 20 in panel b), and between Jan. 20 and 21 in panel c). Negative and positive values represent a de-/increase of energy density, respectively.

from Fig. 8 for the integrated energy density, one finds that $\Delta u_{1}$ and $\Delta u_{2}$ are predominantly positive, especially above a height of $\approx 35^{\prime \prime}(\approx 25 \mathrm{Mm})$. Positive values of $\Delta u_{1}$ and $\Delta u_{2}$ mean that the amount of stored energy in the considered volume increased until Jan. 20. This might be due to the changing magnetic field configuration during the evolution of the upcoming flaring activity. One also finds some small areas of $\Delta u_{1}<0$ in panel (a), especially below $\approx 15^{\prime \prime}(\approx 11 \mathrm{Mm})$, which are continuously decreasing in size with increasing height. In small parts some magnetic energy seems to be moved around, e.g., by flows corresponding to slow surface motions, or even to be related to the weak C-class flaring on Jan. 18. Furthermore, this might be the reason for the absence of a strong gradient in $\Delta e_{1}$ in Fig. 8, showing the change in the integrated energy density as purely positive. However, these small negative regions as visible in panel (a) appear in general to be positive in panel (b); i.e., energy was also now stored at low heights. One should notice that the major increase in energy storage now seems to take place in regions that appeared highly negative in panel (a), which might be related to the footpoints of the flare ribbons as visible in the $\mathrm{H} \alpha$ images described by Goff et al. (2007). Again, the main activity is taking place at low heights $\left(\$ 20^{\prime \prime}\right.$, i.e., $\left.\approx 14 \mathrm{Mm}\right)$, but the location of the energy loss (as indicated by the negative values of $\Delta e_{2}$ in Fig. 8) cannot be defined clearly. In panel (c) of Fig. 9 the change in the energy density appears mostly negative $\left(\Delta u_{3}<0\right.$, in agreement with the purely negative values of $\Delta e_{3}$ in Fig. 8), so the energy density on Jan. 21 was lower than on Jan. $20\left(u_{21}<u_{20}\right)$; i.e., a considerable amount of energy was released during the M6.1 flare on Jan. 20. This might have taken place in the form of converting magnetic energy into other forms of energy by means of, e.g. magnetic reconnection. As found for the previous days, the changes in the amount of stored energy in the considered subvolume take place predominately within a height of $\approx 30^{\prime \prime}$ $(\approx 22 \mathrm{Mm})$ above the photosphere.

\section{Discussion}

We investigated the 3D coronal magnetic field configuration related to the flaring activity of NOAA AR 10540 from Jan. 18 to 21,2004 . By using a multigrid-like optimization code, we 
extrapolated the coronal field from photospheric magnetic field measurements as obtained from the NAOJ/SFT. We calculated the energy content of the extrapolated domain for the four consecutive days and estimated the upper limit for the free magnetic energy available to power two M-class flares recorded during this time period (a M1.0 flare on Jan. 19 and a M6.1 flare on Jan. 20).

We find that the total magnetic energy increases before the M6.1 flare on Jan. 20 and decreases afterwards. Before, as well as after, the flaring activity, the total magnetic energy of the calculated, nonlinear force-free field is approximately $E_{\text {nlff }} \propto$ $10^{26} \mathrm{~J}$, which is also true for the potential field. The expectation that the nonlinear force-free field energy exceeds that of the potential field can be clearly recovered. Besides this, the energy drop of the nonlinear force-free field is higher than that of the potential field; i.e., the energy release seems to be related to the change in the transversal magnetic field rather than the normal component of it. This supports the hypothesis that the flare energy was taken from the coronal magnetic field where it was slowly built up and stored prior to the flare onset. The upper limit of the free magnetic energy available to be set free could be estimated with $E_{\text {free }} \propto 10^{25} \mathrm{~J}$, which is enough to power a large flare, according to Priest \& Forbes (2002). The first, smaller M1.0 flare does not seem to have much influence on the magnetic energy content of the coronal field above AR 10540. This might be due to the low time cadence of the photospheric vector magnetograph data we used since this smaller event may not have been sufficiently resolved in time, and moreover flux was still emerging at this time, hence reducing the effect of the smaller flare, too. The comparison of the nonlinear force-free field and the potential field shows that the ratio of its energy content goes down remarkably after the M6.1 flare but does not seem to be responsive to the M1.0 flare. Moreover, the field does not relax to the potential configuration, since this ratio does not reach unity after the M6.1 flare. In other words, the whole amount of available free magnetic energy is not released during this flare.

We also investigated the evolution of the integrated energy density with height to be able to restrict the area in which the energy changes due to the flaring activity mainly take place. We find that the major changes in the considered subvolume above the flaring region are restricted to a height of $\lesssim 30^{\prime \prime}$, i.e., $\$ 22 \mathrm{Mm}$ above the photosphere, which varies slightly for the consecutive days. The integrated energy density as a function of height is lowest on Jan. 21, which can be interpreted such that, since the M6.1 flare was already launched, previously built-up magnetic energy was released during that process. On all other days, one finds a clearly higher amount of energy storage that can be assigned to the change of the magnetic field configuration leading to the upcoming flare activity, including continuous flux emergence until Jan. 20. The change in the integrated energy density shows us that the absolute values of the integrated energy density decrease with increasing height. However, it cannot be stated that its relative values could be purely assigned to the flaring activity since magnetic flux was still emerging until Jan. 20. Only the difference in the integrated energy density of the last two days turns out to be purely negative, which also can be attributed to the release of free energy during the M6.1 flare. This can also be seen if one investigates the change in the energy density itself, which appears to be predominantly negative within the considered subvolume only after the M6.1 flare.

The sequence of nonlinear force-free equilibria used here has a rather low time cadence of one day. The trend that magnetic energy is quasistatically built up before a flare is, nevertheless, clearly visible. We also noticed an decrease in the energy after the M6.1 flare, but the low time cadence does not allow us to decide which part of this energy drop is related to the sudden release of energy due to the eruptive phenomena or to the simultaneously visible decay of the AR. Vectormagnetogram measurements with a much higher time cadence, as expected in future, e.g., from the SDO/HMI (Graham et al. 2003) or SOLIS/VSM (Keller et al. 2003) instruments, and corresponding coronal field extrapolations might help for investigating the flare mechanism in more detail.

Investigations of the magnetic field topology before and after flares with a high temporal cadence might shed light on the role of magnetic reconnection for flare activity. The special interest would therefore be to distinguish the influence of photospheric and coronal reconnections. One would be able to study in more detail whether the drop in the magnetic energy content during a flare finds a correspondence in the change of the underlying photospheric magnetic field or if such changes may even play an active role during solar flares.

Acknowledgements. We performed the study of AR 10540 with the use of NAOJ/SFT vector magnetograms and MDI LOS magnetic field data of the Solar and Heliospheric Observatory (SoHO).

The work of J. K. Thalmann got financial support by DFG-grant WI 3211/1-1 and T. Wiegelmann was supported by DLR-grant 50 OC 0501.

\section{References}

Alissandrakis, C. E. 1981, A\&A, 100, 197

Aly, J. J. 1989, Sol. Phys., 120, 19

Amari, T., Aly, J. J., Luciani, J. F., Boulmezaoud, T. Z., \& Mikic, Z. 1997, Sol. Phys., 174, 129

Amari, T., Boulmezaoud, T. Z., \& Mikic, Z. 1999, A\&A, 350, 1051

Amari, T., Boulmezaoud, T. Z., \& Aly, J. J. 2006, A\&A, 446, 691

Archontis, V., Moreno-Insertis, F., Galsgaard, K., \& Hood, A. W. 2006, in Solar Activity and its Magnetic Origin, ed. V. Bothmer, \& A. A. Hady, IAU Symp., 233, 53

Barnes, G., \& Leka, K. D. 2006, ApJ, 646, 1303

Bleybel, A., Amari, T., van Driel-Gesztelyi, L., \& Leka, K. D. 2002, A\&A, 395, 685

Chiu, Y. T., \& Hilton, H. H. 1977, ApJ, 212, 873

Fuhrmann, M., Seehafer, N., \& Valori, G. 2007, A\&A, 476, 349

Gary, G. A. 2001, Sol. Phys., 203, 71

Goff, C. P., van Driel-Gesztelyi, L., Démoulin, P., et al. 2007, Sol. Phys., 240, 283

Graham, J. D., Norton, A., López Ariste, A., et al. 2003, in ASP Conf. Ser., ed. J. Trujillo-Bueno, \& J. Sanchez Almeida, 307, 131

Handy, B. N., Acton, L. W., Kankelborg, C. C., et al. 1999, Sol. Phys., 187, 229 Inhester, B., \& Wiegelmann, T. 2006, Sol. Phys., 235, 201

Jing, J., Wiegelmann, T., Suematsu, Y., Kubo, M., \& Wang, H. 2008, ApJ, 676, L81

Keller, C. U., Harvey, J. W., \& Giampapa, M. S. 2003, in Society of PhotoOptical Instrumentation Engineers (SPIE) Conference, Innovative Telescopes and Instrumentation for Solar Astrophysics., ed. S. L. Keil, \& S. V. Avakyan, 4853, 194

Kosovichev, A. G., \& Duvall, T. L. 2006, in Solar Activity and its Magnetic Origin, ed. V. Bothmer, \& A. A. Hady, IAU Symp., 233, 365

Kosovichev, A. G., \& Zharkova, V. V. 1999, Sol. Phys., 190, 459

Kosugi, T., Matsuzaki, K., Sakao, T., et al. 2007, Sol. Phys., 243, 3

Kusano, K., Maeshiro, T., Yokoyama, T., \& Sakurai, T. 2002, ApJ, 577, 501

Lin, H., Kuhn, J. R., \& Coulter, R. 2004, ApJ, 613, L177

Maeshiro, T., Kusano, K., Yokoyama, T., \& Sakurai, T. 2005, ApJ, 620, 1069

Mandrini, C. H., Pohjolainen, S., Dasso, S., et al. 2005, A\&A, 434, 725

Metcalf, T. R., Jiao, L., McClymont, A. N., Canfield, R. C., \& Uitenbroek, H. 1995, ApJ, 439, 474

Metcalf, T. R., Leka, K. D., \& Mickey, D. L. 2005, ApJ, 623, L53

Metcalf, T. R., Derosa, M. L., Schrijver, C. J., et al. 2008, Sol. Phys., 247, 269

Molodenskii, M. M. 1969, SvA, 12, 585

Molodensky, M. M. 1974, Sol. Phys., 39, 393

Nakagawa, Y. 1976, Space Sci. Rev., 19, 459

Neukirch, T. 2005, in Chromospheric and Coronal Magnetic Fields, ed. D. E.

Innes, A. Lagg, \& S. A. Solanki, ESA Special Publication, 596

Nindos, A., \& Andrews, M. D. 2004, ApJ, 616, L175 
Nindos, A., Zhang, J., \& Zhang, H. 2003, ApJ, 594, 1033

Ogawara, Y., Takano, T., Kato, T., et al. 1991, Sol. Phys., 136, 1

Priest, E. R., \& Forbes, T. G. 2002, A\&A Rev., 10, 313

Régnier, S., \& Amari, T. 2004, A\&A, 425, 345

Régnier, S., \& Canfield, R. C. 2006, A\&A, 451, 319

Régnier, S., \& Priest, E. R. 2007a, ApJ, 669, L53

Régnier, S., \& Priest, E. R. 2007b, A\&A, 468, 701

Régnier, S., Amari, T., \& Kersalé, E. 2002, A\&A, 392, 1119

Régnier, S., Fleck, B., Abramenko, V., \& Zhang, H.-Q. 2005, in Chromospheric and Coronal Magnetic Fields, ed. D. E. Innes, A. Lagg, \& S. A. Solanki, ESA Special Publication, 596

Sakurai, T. 1981, Sol. Phys., 69, 343

Sakurai, T. 1982, Sol. Phys., 76, 301

Sakurai, T., Ichimoto, K., Nishino, Y., et al. 1995, PASJ, 47, 81

Scherrer, P. H., Bogart, R. S., Bush, R. I., et al. 1995, Sol. Phys., 162, 129

Schmidt, H. U. 1964, in The Physics of Solar Flares, ed. W. N. Hess, 107

Schrijver, C. J., Derosa, M. L., Metcalf, T. R., et al. 2006, Sol. Phys., 235, 161

Schrijver, C. J., DeRosa, M. L., Metcalf, T., et al. 2008, ApJ, 675, 1637

Seehafer, N. 1978, Sol. Phys., 58, 215

Solanki, S. K., Lagg, A., Woch, J., Krupp, N., \& Collados, M. 2003, Nature, 425, 692

Somov, B. V., Kosugi, T., Hudson, H. S., Sakao, T., \& Masuda, S. 2002, ApJ, 579,863

Song, M. T., Fang, C., Tang, Y. H., Wu, S. T., \& Zhang, Y. A. 2006, ApJ, 649, 1084

Tang, Y. H., Li, Y. N., Fang, C., et al. 2000, ApJ, 534, 482
Tsuneta, S., Acton, L., Bruner, M., et al. 1991, Sol. Phys., 136, 37

Valori, G., Kliem, B., \& Keppens, R. 2005, A\&A, 433, 335

Valori, G., Kliem, B., \& Fuhrmann, M. 2007, Sol. Phys., 245, 263

van Driel-Gesztelyi, L., Manoharan, P. K., Démoulin, P., et al. 2000, J. Atm. Solar-Terrestrial Phys., 62, 1437

Wheatland, M. S. 2004, Sol. Phys., 222, 247

Wheatland, M. S. 2006, Sol. Phys., 238, 29

Wheatland, M. S. 2007, Sol. Phys., 245, 251

Wheatland, M. S., Sturrock, P. A., \& Roumeliotis, G. 2000, ApJ, 540, 1150

Wiegelmann, T. 2004, Sol. Phys., 219, 87

Wiegelmann, T. 2007, Sol. Phys., 240, 227

Wiegelmann, T. 2008, J. Geophys. Res. (Space Physics), 113, 3

Wiegelmann, T., \& Neukirch, T. 2003, Nonlinear Processes in Geophysics, 10, 313

Wiegelmann, T., Inhester, B., Lagg, A., \& Solanki, S. K. 2005a, Sol. Phys., 228, 67

Wiegelmann, T., Lagg, A., Solanki, S. K., Inhester, B., \& Woch, J. 2005b, A\&A, 433, 701

Wiegelmann, T., Inhester, B., Kliem, B., Valori, G., \& Neukirch, T. 2006a, A\&A, 453,737

Wiegelmann, T., Inhester, B., \& Sakurai, T. 2006b, Sol. Phys., 233, 215

Wu, S. T., Sun, M. T., Chang, H. M., Hagyard, M. J., \& Gary, G. A. 1990, ApJ, 362, 698

Yamamoto, T. T., Kusano, K., Maeshiro, T., Yokoyama, T., \& Sakurai, T. 2005, ApJ, 624, 1072

Yan, Y., \& Li, Z. 2006, ApJ, 638, 1162 